\title{
Broken space-time symmetries and mechanisms of rectification of ac fields by nonlinear (non)adiabatic response
}

Denisov, S.; Flach, S.; Ovchinnikov, A. A.; Yevtushenko, O.; Zolotaryuk, Yaroslav

Published in:

Physical Review E. Statistical, Nonlinear, and Soft Matter Physics

Link to article, DOI:

10.1103/PhysRevE.66.041104

Publication date:

2002

Document Version

Publisher's PDF, also known as Version of record

Link back to DTU Orbit

Citation (APA):

Denisov, S., Flach, S., Ovchinnikov, A. A., Yevtushenko, O., \& Zolotaryuk, Y. (2002). Broken space-time symmetries and mechanisms of rectification of ac fields by nonlinear (non)adiabatic response. Physical Review E. Statistical, Nonlinear, and Soft Matter Physics, 66(4), 041104. https://doi.org/10.1103/PhysRevE.66.041104

\section{General rights}

Copyright and moral rights for the publications made accessible in the public portal are retained by the authors and/or other copyright owners and it is a condition of accessing publications that users recognise and abide by the legal requirements associated with these rights.

- Users may download and print one copy of any publication from the public portal for the purpose of private study or research.

- You may not further distribute the material or use it for any profit-making activity or commercial gain

- You may freely distribute the URL identifying the publication in the public portal 


\title{
Broken space-time symmetries and mechanisms of rectification of ac fields by nonlinear (non)adiabatic response
}

\author{
S. Denisov, ${ }^{1}$ S. Flach, ${ }^{2}$ A. A. Ovchinnikov, ${ }^{2}$ O. Yevtushenko, ${ }^{3}$ and Y. Zolotaryuk ${ }^{4,5}$ \\ ${ }^{1}$ Department of Chemistry, Tel-Aviv Unversity, Tel-Aviv 69978, Israel \\ ${ }^{2}$ Max-Planck-Institut für Physik komplexer Systeme, D-01187 Dresden, Germany \\ ${ }^{3}$ Abdus Salam ICTP, 34100 Trieste, Italy \\ ${ }^{4}$ Section of Mathematical Physics, IMM, Technical University of Denmark, Lyngby 2800, Denmark \\ ${ }^{5}$ Bogolyubov Institute for Theoretical Physics, National Academy of Sciences of Ukraine, Kiev 03143, Ukraine
}

(Received 12 April 2002; published 10 October 2002)

\begin{abstract}
We consider low-dimensional dynamical systems exposed to a heat bath and to additional ac fields. The presence of these ac fields may lead to a breaking of certain spatial or temporal symmetries, which in turn cause nonzero averages of relevant observables. Nonlinear (non)adiabatic response is employed to explain the effect. We consider a case of a particle in a periodic potential as an example and discuss the relevant symmetry breakings and the mechanisms of rectification of the current in such a system.
\end{abstract}

DOI: 10.1103/PhysRevE.66.041104

PACS number(s): 05.60.Cd, 05.45.Ac

\section{INTRODUCTION}

Much has been written on noise induced transport, where noise may be colored, or simply white with a time periodic signal. Arguments include violation of fluctuation-dissipation theorems, breaking of reflection symmetries of potentials in space, Maxwell's demons, mixing of harmonics (of, e.g., a periodic drive), etc. All this applies to both classical and quantum systems, and extends at least conceptually to stochastic resonance, quantum stochastic resonance, etc. A recent review on ratchet transport by Reimann [1] provides a lot of theoretical and experimental results, and we refer the interested reader to this work.

For a set of related problems, such as directed particle current $[2,3]$, directed energy current [4], average magnetization [5], and nonlinear Hall effect [6], to name a few, a recently published symmetry approach was shown to systematically account for all relevant symmetries that have to be broken in order to explain the observed rectification effect. The purpose of this paper is to generalize this approach, and to apply it to different physically relevant situations such as underdamped, overdamped, or zero temperature cases. We also use the nonlinear response arguments to make the results of symmetry considerations very transparent. We will argue for a rather minor role of additional fluctuations that are mainly responsible for the area of phase space explored. Although we will discuss mostly the case of time-periodic external fields, we will also show how the nonlinear response concept can be generalized to fields that are quasiperiodic in time.

The paper has the following structure. In the following section we discuss the symmetry breaking by considering the adiabatic limit and making use of some general forms of nonlinear response functions. Section III is devoted to the detailed symmetry analysis of a classical particle moving in a periodic potential under the influence of an external ac drive. Symmetry breaking leads to a directed current here. We will discuss the presence of ballistic channels which provide a mechanism of current rectification. We will show that these ballistic channels survive in the presence of dissipation. Section IV addresses the case of a particle with nonparabolic dispersion. The addition of a spatial potential allows us to tune the system in such a way that ballistic channels disappear. This will lead to an expected dramatic drop of the directed current value. Conclusions and discussions are given in Sec. V.

\section{ADIABATIC RESPONSE TO SLOW ac FIELDS}

Consider a certain system in contact with a heat bath. The system is characterized by some internal (nonlinear) dynamics, and we will discuss concrete models below. So far we just need to know that its state can be characterized by certain variables that are functions of time. These could be functions of phase space variables of a classical system, or expectation values of operators for a quantum system. Consider one of such variables which we denote as $A(t)$. The coupling to the heat bath will be characterized by at least two parameters - the temperature of the bath (we will use the notation of inverse temperature $\beta$ ) and some set of relaxation times, in the simplest case just one relaxation time (we will use the notation of the inverse relaxation time $\nu$ ). We also assume that the chosen variable $A(t)$ is zero on average.

Let us now apply a static field $E$ which couples to $A$ such that a nonzero average $\widetilde{A}=\langle A(t)\rangle_{t}$ is generated. Its dependence on $E$ is assumed to be given by some single valued response function $\widetilde{A}=f(E)$ [7] (the physical meaning of the response function can be different, electric polarization or magnetization, for example). The above mentioned symmetries will be connected to corresponding symmetries of the function $f(E)$. This function will be in general nonlinear, yet in some cases it may be expanded in a Taylor series around $E=0$ and start with a linear term (this term describes then the linear response). There are two possibilities: the function $f(E)$ is either antisymmetric $f(E)=-f(-E)$ or it is asymmetric $f(E) \neq-f(-E)$.

Before analyzing the case of adiabatically slow periodic ac fields, we will more rigorously introduce the notion of 
possible symmetries of an arbitrary periodic function with zero mean.

\section{A. Classification of symmetries of a periodic function with zero mean}

Consider a periodic function $g(z+2 \pi)=g(z)$ having zero mean. First, such functions may be symmetric around certain $z$ values. Without loss of generality, this point may be set to zero and we find $g(z)=g(-z)$. We will use the abbreviation $g_{s}$ in such a case. Second, such functions may be antisymmetric $g(z)=-g(-z)$ (abbrevation $g_{a}$ ). Note that the points around which a function is symmetric and antisymmetric will be different if the function possesses both symmetries. Finally, the function may possess shift symmetry (which is also called antiperiodicity): $g(z+\pi)=-g(z)$ (abbreviation $g_{s h}$ ). A zero mean periodic function may possess none of the above symemtries, precisely one of them, or all three simultaneously. In particular, $g_{s}$ functions may be expanded in a pure cosine Fourier series, $g_{a}$ functions in a pure sine Fourier series, and $g_{s h}$ functions show up with zero even Fourier components in their Fourier series expansion. As a consequence, the simplest function $g(z)=\cos \left(z+z_{0}\right)$ possesses all three symmetries. The function $g(z)=\cos (z)$ $+\cos \left(2 z+z_{0}\right)$ does not possess any of the listed symmetries except for $z_{0}=0, \pi\left(g_{s}\right)$, and $z_{0}= \pm \pi / 2\left(g_{a}\right)$. A final example $g(z)=\cos (z)+\cos \left(3 z+z_{0}\right)$ always possesses shift symmetry $\left(g_{s h}\right)$ and in addition may be symmetric and antisymmetric for $z_{0}=0, \pi$.

Let us finally note that most cases under consideration use several harmonics contained in the drive. However, it may be also important to use pulse sequences. The symmetry considerations can be straightforwardly applied also to such cases.

\section{B. Periodic ac fields}

Let us now assume that the field $E$ is slowly varying in time. Slowly means that the characteristic time scales of changes of $E(t)$ are much larger than all other time scales in the system. Then we are dealing with the adiabatic limit of the response to time-dependent fields. We may use the response $f$ to a static field and simply insert the slow time dependence: $f(E(t))$. Consider a time-periodic field $E(t)$ $=E(t+T)$ with zero average $\widetilde{E}=0$. In general, the corresponding value $\widetilde{A}=1 / T \int f(E(t)) d t$ will be nonzero for asymmetric $f$. However, for antisymmetric single valued $f$ the average $\widetilde{A}$ will be zero provided the function $E(t)$ is either antisymmetric $E(t)=-E(-t)$ or has shift symmetry $E(t)$ $=-E(t+T / 2)$.

Consider as an example

$$
E(t)=E_{1} \cos t+E_{2} \cos (2 t+\alpha)+E_{3} \sin \left(3 t+\alpha^{\prime}\right) .
$$

For $E_{2}=E_{3}=0$ Eq. (1) has all three mentioned symmetries of periodic functions. For $E_{3}=0$ the function has no symmetries except for $\alpha=n \pi$ ( $n$ integer) where it is symmetric, or for $\alpha=\pi(1 / 2+n)$ where the function is antisymmetric. For $E_{2}=0$ Eq. (1) has shift symmetry except for $\alpha^{\prime}=\pi(1 / 2$ $+n)$ when it is in addition symmetric and antisymmetric (around different time origins, of course).

Next we assume a case when the adiabatic response function $f$ may be expanded in a Taylor series,

$$
f(E)=f_{1} E+f_{2} E^{2}+f_{3} E^{3}+\cdots,
$$

where the skipping of higher order terms is justified by the smallness of $E$. For $f_{2} \neq 0$ the response is asymmetric and consequently any field from Eq. (1) will in general lead to a nonzero average of $f$. For $f_{2}=0$, however, all shift symmetric and antisymmetric $E(t)$ will yield zero averaged $f$ (because it leads also to a shift symmetry or antisymmetry of $f$ in time). As an example of mixing of harmonics the leading nonzero contribution for $E_{2} \neq 0, E_{3}=0$ equals

$$
\widetilde{f}=\frac{1}{T} \int_{0}^{T} f(E(t)) d t=\frac{3}{4} f_{3} E_{1}^{2} E_{2} \cos \alpha+\cdots .
$$

In accordance with the above said, $\widetilde{f}$ vanishes for $\alpha$ $=\pi(1 / 2+n)$. Note that the antisymmetry property of $f(E)$ is linked to some internal symmetries of the dynamical system under consideration, as will be shown below.

It is important to note that the above is valid for single valued response functions. If the coupling to a heat bath is too weak, response functions may contain hysteretic loops. Such cases need separate discussion [7].

\section{Quasiperiodic ac fields}

We may even consider quasiperiodic driving here. Suppose that we drive the system with a field $E(t)=e_{1}(t)$ $+e_{2}(t)$ where both components $e_{1,2}$ are time periodic, but with incommensurable periods. The resulting field $E(t)$ would be then quasiperiodic. If considering low amplitude fields and expanding the response function to second or third order, the full dc average of $A(t)$ will be given by a mere sum of the averages obtained in the presence of only one of the two periodic fields. Consider, e.g., $E(t)=e_{1} \cos \omega_{1} t$ $+e_{2} \cos \omega_{2} t$ and an asymmetric response. In lowest order in the field amplitudes we obtain

$$
\tilde{f}=f_{2}\left(\frac{1}{2} e_{1}^{2}+\frac{1}{2} e_{2}^{2}\right)+f_{4}\left(\frac{3}{8} e_{1}^{4}+\frac{3}{8} e_{2}^{4}+\frac{6}{4} e_{1}^{2} e_{2}^{2}\right)+\cdots .
$$

In higher orders in the response interference effects appear, so that products of the field components enter the result. Yet for the lowest order case $\left(f_{2}\right)$ only the sum appears. So we may assume that further changing the drive by adding more time periodic field components does not change the main result of a possibility of a nonzero average.

If we consider an antisymmetric response function $f$, we need to choose a more sophisticated quasiperiodic drive. Take, e.g., $\quad E(t)=e_{1}(t)+e_{2}(t) \quad$ with $\quad e_{i}(t)=e_{i 1} \cos \omega_{i} t$ $+e_{i 2} \cos \left(2 \omega_{i} t+\alpha_{i}\right)$. Assuming again that the field amplitude is small, we obtain in lowest order

$$
\widetilde{f}=\frac{3}{4} f_{3}\left(e_{11}^{2} e_{12} \cos \alpha_{1}+e_{21}^{2} e_{22} \cos \alpha_{2}\right) .
$$


Again we find that the periodic components of the quasiperiodic drive contribute additively in lowest order. Note that each contribution can be obtained by considering a reduced drive function consisting only of this part. The corresponding contribution is clearly related to the symmetry properties of this reduced drive.

\section{Do we need more?}

Once a given problem is considered in the adiabatic limit like discussed above, the appearance of nonzero averages due to ac fields can be obtained, and further changing of parameters away from the adiabatic limit will change numbers, but not the fact of nonzero averages. Ratchet transport in its general form falls into such a class of systems. Indeed a particle moving in a periodic potential $V(x)=V(x+\lambda)$ under the influence of an external colored noise and dissipation may be taken into the limit of zero temperature (no noise), slow periodic driving, and zero mass (overdamped case),

$$
\dot{x}+V^{\prime}(x)+E(t)=0 .
$$

The response of $\langle\dot{x}\rangle$ to a corresponding static field $E$ is well known and has been discussed in connection with Josephson current-voltage characteristics (for symmetric potentials). The response is clearly nonlinear, and antisymmetric if the potential is symmetric in space. In case of asymmetric ratchet potentials the response is nonlinear but asymmetric. In the latter case a simple $E(t) \sim \cos t$ signal with large enough amplitude will generate a nonzero averaged current. In case of symmetric potentials a drive which is neither antisymmetric nor shift symmetric is needed.

Nonadiabatic corrections are of interest and importance. For example, upon constantly increasing the frequency of a drive, currents (or other averages) may change sign, or increase or decrease by orders of magnitude [8-10]. Especially interesting are cases when certain (nonadiabatic) parameter limits give rise to new symmetries. These symmetries may be traced back away from the corresponding limits and help understand lots of peculiar features that are observed in such driven systems.

If the adiabatic response simply vanishes, nonadiabatic response contributions will be not just mere corrections. In such cases the symmetry considerations of the underlying equations of motion presented below appear to be the most direct way to predict rectification effects. One example of such a case is given in Ref. [5] where the problem of driven quantum spins in ac magnetic fields is considered. While the adiabatic limit provides with zero induced magnetization component in $y$ direction there, nonadiabatic response terms make this component nonzero, as expected from symmetry considerations.

\section{DIRECTED CURRENTS: THE CASE OF A CLASSICAL PARTICLE IN A PERIODIC POTENTIAL}

In order to make further progress in the understanding of rectification it is useful to define a model. Here we will consider the case of a particle of the unit mass moving in a space-periodic potential $V(x)=V(x+\lambda)$ under the influence of friction and external forces,

$$
m \ddot{x}+\gamma \dot{x}-f(x)-\chi(t)=0,
$$

where $f(x)=-V^{\prime}(x)$ and $\chi(t)$ is zero on average. The mass $m$ is assumed to be equal to one if not stated otherwise. If $\chi(t)$ is a Gaussian white noise, the particle undertakes diffusion with zero net current, in accord with the fluctuationdissipation theorem. If, however, $\chi(t)$ contains correlations (color), then it is known that a nonzero net (dc) current is possible. In order to understand this result, it is appropriate to make the correlations in the noise as transparent as possible. The easiest way to do so is to choose

$$
\chi(t)=\xi(t)+E(t), \quad E(t)=E(t+T),
$$

where $\xi(t)$ is a Gaussian white noise and the time-periodic external field $E(t)$ has zero mean. A probabilistic description of the system is then possible using a Fokker-Planck equation (see Appendix).

In a next radical step we skip the $\xi(t)$ term. The reason for this is that we will be left with a deterministic equation, whose symmetries may be studied. These symmetries involving operations in time $t$ are assumed to hold even in the presence of a Gaussian white noise, since such a term does not contain temporal correlations. Note that the general nonintegrability of the resulting equations may provide with irregular (chaotic) trajectories. Thus we are left with an equation of the form

$$
m \ddot{x}+\gamma \dot{x}-f(x)-E(t)=0 .
$$

\section{A. The relevant symmetries and ways of violations}

In order to characterize the symmetries of Eq. (9) we remind that the phase space dimension is three. As we look for nonzero average currents which are characterized by the velocity $\dot{x}$, we have to check whether there exist symmetries that allow to generate out of a specific trajectory of Eq. (9) another one with reversed velocities. The transformations of interest have to involve a change of sign of $\dot{x}$ leaving its absolute value unchanged. Thus we look for transformations that leave Eq. (9) invariant and (a) either change the sign of $x \rightarrow-x$ and simultaneously shift time $t \rightarrow t+t_{0}$ or (b) invert time $t \rightarrow-t$ and shift coordinate $x \rightarrow x+x_{0}$.

The following symmetries can be identified $[2,3]$ :

$$
\begin{gathered}
\hat{S}_{a}, \quad x \rightarrow-x, t \rightarrow t+\frac{T}{2}, \text { if }\left\{f_{a}, E_{s h}\right\}, \\
\hat{S}_{b}, \quad x \rightarrow x, \quad t \rightarrow-t, \quad \text { if }\left\{E_{s}, \gamma=0\right\}, \\
\hat{S}_{c}, \quad x \rightarrow x+\frac{\lambda}{2}, \quad t \rightarrow-t \quad \text { if } \quad\left\{f_{s h}, E_{a}, m=0\right\} .
\end{gathered}
$$

Note that $\hat{S}_{b}$ and $\hat{S}_{c}$ require $\gamma=0$ (Hamiltonian case) and $m=0$ (overdamped case), respectively. Another observation is that all symmetries require certain symmetry properties of the external drive $E(t)$, while the properties of the space- 
periodic force $f$ not matter for $\hat{S}_{b}$. A proper choice of the drive may thus break all of the above listed symmetries with any coordinate dependence of the force $f(x)$.

It is useful to provide an interpretation of the action of the above symmetry operations on trajectories of Eq. (9). If the equation of motion is invariant under a symmetry, it implies that a given solution or trajectory, when transformed using the symmetry operation, yields again a solution or trajectory of the system. It may be the same trajectory or a different one. The symmetry $\hat{S}_{a}$ for $\gamma \neq 0$ transforms an attractor into an attractor, repellers, into repellers and basins of attraction into basins of attraction. Thus if an attractor is mapped onto itself, the average velocity on that attractor is zero. If an attractor is mapped onto another attractor, we find complete symmetry between the two attractors and their basins of attraction, while the average velocities will be of opposite sign. The symmetry $\hat{S}_{b}$, which is valid for the Hamiltonian case, simply relates two trajectories to each other, both having opposite average velocities.

Once we add white noise, we have to consider some way of weighting different trajectories. In that sense, if two different trajectories are related by a symmetry $\hat{S}_{a}$ or $\hat{S}_{b}$ and have identical statistical weight, their contributions to a total average current will annihilate.

The symmetry $\hat{S}_{c}$ is acting in the overdamped limit, yet it is reverting the sign of time. Thus it maps an attractor on a repeller and the basin of attraction of an attractor into the basin of repulsion of a repeller. It follows that due to the low dimension $d=2$ of the phase space of this problem, the compactness of the phase space, and the uniqueness of a trajectory running through a point in it, all trajectories have zero average velocity when $\hat{S}_{c}$ is valid.

In order to correctly incorporate the effects of noise, kinetic equations have to be considered. This is done in Appendix.

If at least one of the symmetries (10) is valid, we conclude that the averaged current is zero if an additional white Gaussian noise term is added. This noise will simply lead to an exploration of the whole phase space and thus realize on finite times different trajectories of the deterministic system.

Once all of the above symmetries are broken, we may expect that in general the average current will be nonzero. The understanding of the mechanisms that will lead to this current can be best obtained in the absence of noise for the deterministic system.

\section{B. Rectification mechanisms in the Hamiltonian limit}

As it was shown in Ref. [3], the value of the average current is strongly enhanced for the underdamped case, when the dissipation rate tends to zero. Thus we start our study of the mechanisms with the dissipationless case $\gamma=0$. This Hamiltonian limit of Eq. (7) is generically characterized by nonintegrable dynamics with a mixed phase space containing both chaotic and regular areas [11]. A stochastic layer appears around the separatrix of the integrable nondriven $[E(t)=0]$, Eq. (7).

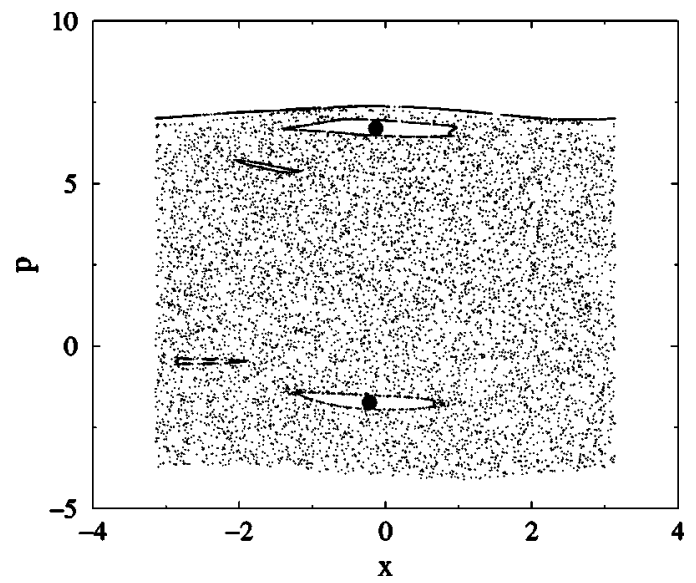

FIG. 1. Poincare map of the main stochastic layer of Eq. (9) with $\gamma=0$ and functions (11) with $E_{1}=3.26, E_{2}=1.2, \alpha=\pi / 2$, and $\omega=1$. The two filled circles correspond to the two observed limit cycle attractors in the weakly dissipative case with damping $\gamma$ $=10^{-4}$.

Contributions to a nonzero current may come from trajectories inside the stochastic layer as well as from regular unbound motion. Because the latter always exist for motion in both directions, strong effective cancellation of currents takes place [2], while the strongest remaining contribution again comes from the stochastic layer, which will be considered below.

In the following we choose the following functions and parameters:

$$
\begin{gathered}
V(x)=-\cos x, \\
E(t)=E_{1} \cos \omega t+E_{2} \cos (2 \omega t+\alpha) .
\end{gathered}
$$

This choice ensures that the symmetries $\hat{S}_{a}$ and $\hat{S}_{b}$ are broken. In Fig. 1 we show the Poincare map of the main stochastic layer of our model.

Points with coordinates $p=\dot{x}$ and $x \bmod 2 \pi$ are drawn after each period of the drive. In Fig. 2 the time dependence of the coordinate $x(t)$ is shown.

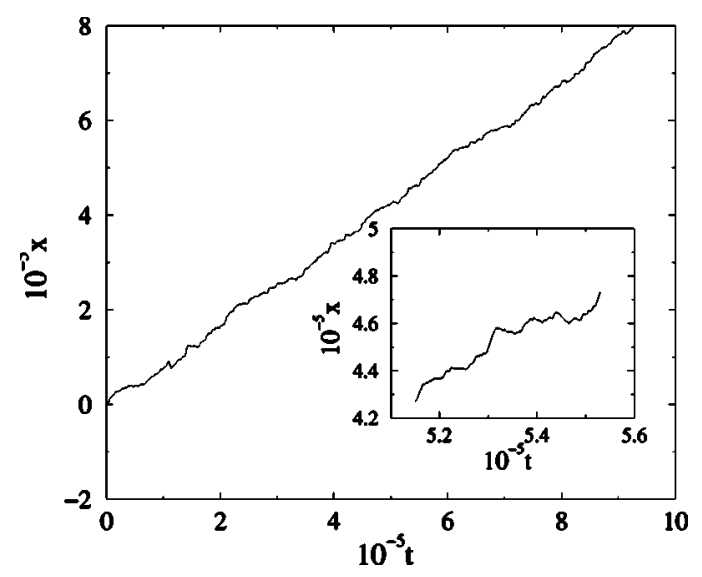

FIG. 2. $x(t)$ of trajectory from Fig. 1. Inset: enlargement of $x(t)$. Note the axis scales. 


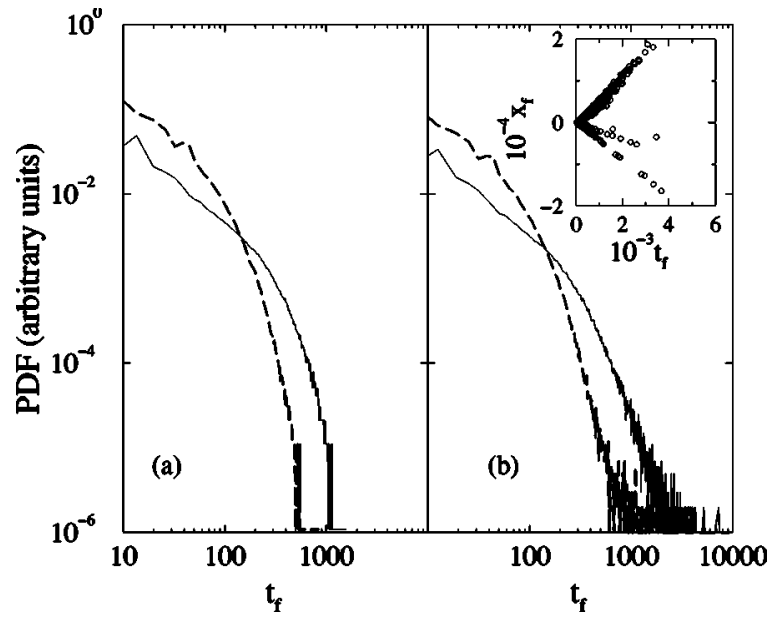

FIG. 3. Probability distributions of ballistic flights to the right (solid line) and left (dashed line). (a) Case with dissipation and noise. (b) Hamiltonian case. Inset: length of ballistic flight vs time of ballistic flight for Hamiltonian case.

We observe a drift in accordance with the symmetry breaking [2]. The average velocity is approximately $\langle\dot{x}\rangle$ $\approx 0.85$.

In order to understand the dynamical mechanisms of a nonzero dc current in the stochastic layer, we first note that the stochastic layer is bounded in phase space. The boundary contains of a fractal set of regular islands embedded in the stochastic layer. A trajectory from the stochastic layer may become trapped for quite long times in these boundaries and perform ballisticlike (regularlike) dynamics. The symmetry breaking of the equations of motion is reflected in a desymmetrization of these fractal boundary structures for the upper and lower boundaries. This in turn leads to a desymmetrization of distribution functions which characterize the probability to stick and stay in such a boundary region [12]. In Fig. 3(b) the probability distribution functions of the flight durations to the left and the right (for technical details see Ref. [12]) are shown. These functions are characterized by algebaically decaying tails, and we clearly observe the above mentioned desymmetrization.

In the inset of Fig. 3(b) each point denotes the distance covered in a given flight during the time of ballisticlike motion. We observe a fine structure with three branches. Two major ones correspond to the main ballistic channels with opposite velocities while a minor third one corresponds to a channel with smaller negative velocity.

\section{Presence of dissipation}

When dissipation is present in the system (i.e., $\gamma \neq 0$ ), the phase space of the system separates into basins of attraction of different low-dimensional attractors. Close to the Hamiltonian limit these attractors are limit cycles: $x(t+T)=x(t)$ $+2 \pi m, \dot{x}(t+T)=\dot{x}(t), m \in \mathbb{Z}$. These limit cycles are locked to the external periodic drive $E(t)$, therefore their period $T$ is characterized by $T=2 n \pi / \omega, n \in \mathbb{Z}$. The average velocity on the limit is given by

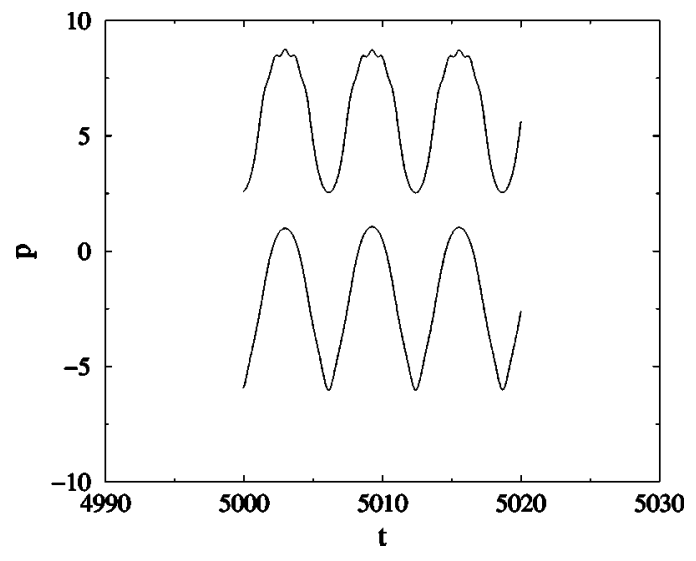

FIG. 4. $p(t)=\dot{x}(t)$ of both attractors for $\gamma=10^{-4}$ which correspond to the two filled circles in Fig. 1.

$$
\langle\dot{x}\rangle=\frac{1}{T} \int_{0}^{T} \dot{x} d t=\frac{m}{n} \omega
$$

When further away from the Hamiltonian, limit chaotic attractors can appear via period-doubling bifurcations [13].

A numerical test reveals that in the case of $\gamma=10^{-4}$ only two limit cycle attractors appear. Their location in the Poincaré map is shown in Fig. 1 by two filled circles. The dependence of $\dot{x}(t)$ on both attractors is shown in Fig. 4 .

Note that the attractors are located inside regular islands of the corresponding nondissipative system. These islands are characterized with nonzero winding numbers, and the sticking of the chaotic trajectory of the nondissipative case provides with the above discussed ballistic channels there. So we observe that the ballistic channels of the Hamiltonian system survive by transforming into limit cycle attractors of the weakly dissipative case.

As the attractors are located inside the stochastic layer of the Hamiltonian limit, their basins of attraction are expected to show up with a complex folding structure. This fact is manifested beautifully if we add a noise of weak intensity which corresponds to a small temperature $1 / \beta=0.05$,

$$
\left\langle\xi(t) \xi\left(t^{\prime}\right)\right\rangle=2 \gamma / \beta \delta\left(t-t^{\prime}\right) .
$$

$1 / \beta$ is small compared to the energy barrier of the periodic potential. As it turns out, in this case of strong external driving the system is so far from the equilibrium case that different scales have to be used in order to compare with the noise intensity. In Fig. 5 we show the corresponding Poincare map over a total time of $t_{f}=10^{6}$. We observe that the trajectory is sticking for long times to the two attractors. But most importantly we observe frequent escapes from the attractors basins. Once the trajectory is kicked out of such a basin, it starts to quickly explore the stochastic layer space, due to the weak damping, weak noise, and the above mentioned complex folding of the basin boundaries. This results in the fact that the probability distribution of the velocities is far from being Maxwellian.

The dependence of $x(t)$ for the dissipative case with noise is shown in Fig. 6. Although the dc current value has 


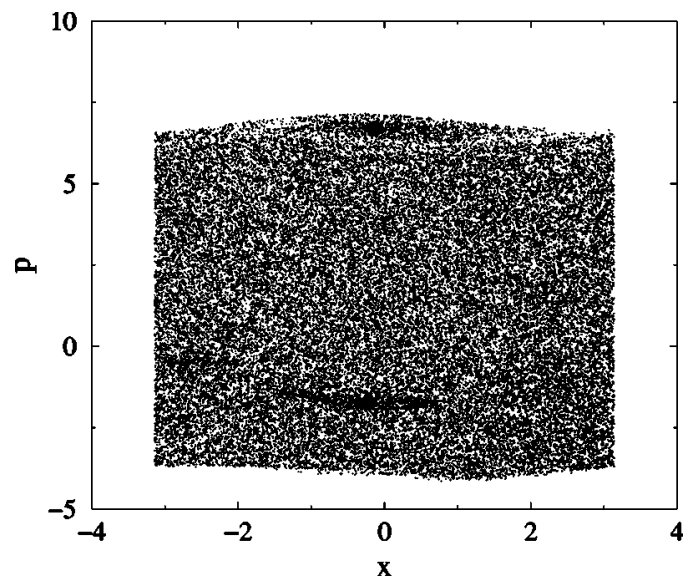

FIG. 5. Poincare map of the dissipative case with weak noise. The two filled circles correspond to the two observed limit cycle attractors for $\gamma=10^{-4}$.

changed compared to the Hamiltonian case, again the evolution is characterized by sticking to ballistic channels.

An evaluation of the corresponding ballistic flight time distributions is shown in Fig. 3(a). A remarkable similarity to the case of the distributions for the Hamiltonian case is observed. Also we observe that the power law tails of the Hamiltonian case are replaced by exponential ones in the dissipative case with noise, due to an expected noise-induced cutoff in the maximum correlation time.

A consequence of the above results is that the loss of ballistic channels due to the variation of some parameter may lead to a crossoverlike decrease of the current. In the following section we will design a model that does possess these properties.

\section{DIRECTED CURRENTS: THE CASE OF NONPARABOLIC DISPERSION}

So far we have discussed the case of a classical particle with parabolic dispersion, i.e., a kinetic energy quadratic in the momentum $p$. What happens if we consider nonparabolic dispersion? A prominent example would be a periodic depen-

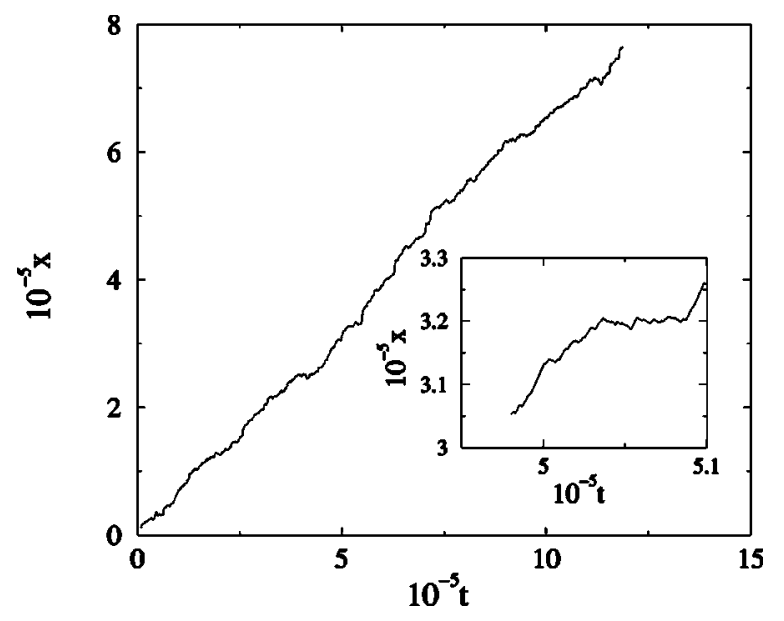

FIG. 6. $x(t)$ of trajectory from Fig. 5. Inset: enlargement of $x(t)$. Note the axis scales. dence on $p$ as for $(-\cos p)$, which reminds the consideration of a quantum particle evolution in one band approximations.

\section{A. Absence of additional potential}

Let us first consider the case of a particle with a general dispersion relation in the presence of additional ac driving,

$$
H=\epsilon(p)-x E(t), \epsilon(p)=\epsilon(-p) .
$$

If the function $\epsilon(p)$ is chosen to be periodic, its period is defined as $\lambda_{p}$. Let us first consider the symmetries of the equations of motion which change the sign of the velocity $\dot{x}$,

$$
\dot{x}=\epsilon^{\prime}(p), \quad \dot{p}=E(t) .
$$

The following symmetries can be identified:

$$
\begin{gathered}
x \rightarrow-x, \quad p \rightarrow-p, \quad t \rightarrow t+\frac{T}{2} \text { if }\left\{E_{s h}\right\}, \\
t \rightarrow-t, \quad p \rightarrow-p \text { if }\left\{E_{s}\right\}, \\
x \rightarrow-x, \quad p \rightarrow p+\frac{\lambda_{p}}{2} \text { if }\left\{\epsilon_{s h}^{\prime}\right\}, \\
t \rightarrow-t, \quad p \rightarrow p+\frac{\lambda_{p}}{2} \text { if }\left\{\epsilon_{s h}^{\prime}, E_{a}\right\} .
\end{gathered}
$$

Note that the last two operations (18), (19) may apply only for periodic $\epsilon(p)$ functions. Furthermore, these operations change the energy of the undriven system. If these operations connect different trajectories, they should not matter at finite temperatures since different energies contribute with different weights.

Choosing the free particle case $\epsilon(p)=p^{2} / 2$ we may arrive at the conclusion that both relevant symmetries (16), (17) can be violated by a proper choice of $E(t)$. Yet, as shown in Refs. [2,14], the expected dc current should be zero in such a case, in fact independent of the strength of some additionally applied dissipation and noise. The case of $\epsilon(p)=-\cos p$ is more involved. On one side it is well known that the kinetic Boltzmann equation solution provides with a nonzero dc current if both symmetries (16), (17) are broken [3]. On the other side this dc current tends to zero as the dissipationless Hamiltonian case is approached. We will show in the following that the reason for that is an additional symmetry of the solutions of Eq. (15) due to the integrability of Eq. (14).

Indeed, integration of Eq. (15) yields

$$
p(t)=g\left(t ; p_{0}, t_{0}\right)=p_{0}+E_{I}(t)-E_{I}\left(t_{0}\right),
$$

where $\dot{E}_{I}(t)=E(t)$. The function $g$ has the property

$$
g(x ; y, z)=-g(z ;-y, x) .
$$

This symmetry operation relates points on a given trajectory to points on a set of other trajectories. Such a symmetry cannot be derived from the equations of motion. It rather is a result of the integrability and reflects the symmetry of the 
solutions, i.e., of the phase space flow (in contrast symmetries of equations of motion relate two trajectories to each other).

The symmetry (21) is in place independent of the choice of the functions $\epsilon(p)$ and $E(t)$ and provides with the following consequences. If we consider a corresponding kinetic equation with finite dissipation, the loss of correlations implies an averaging over the initial phase $t_{0}$ of the field $E(t)$. This averaging persists in the dissipationless limit, while on the other side we recover the above considered Hamiltonian properties. The averaging over $t_{0}$ leads to an enforcement of the symmetry (21), which in turn by changing the sign of $p$ changes the sign of $\dot{x}$. Thus a vanishing dc current is expected in that limit, in accordance with numerical and analytical analysis of kinetic equations. This result can also be obtained from Eqs. (20) and (15) if an averaging with a distribution function, which is symmetric in $p_{0}$ and independent of $x$ and $t_{0}$, is performed,

$$
\int_{0}^{T} d t \int_{0}^{T} d t_{0} \dot{x}\left|p_{0}+\int_{0}^{T} d t \int_{0}^{T} d t_{0} \dot{x}\right|_{-p_{0}}=0 .
$$

To detect a dc current carried by the electrons in a single band one has to break the integrability of the semiclassical equations of motion. We consider this case in the next section. An alternative way is a much more careful treatment of the quantum mechanical problem (see, for example, Ref. [15]), which is, however, far beyond the scope of the present paper.

\section{B. Presence of additional potential}

Things change drastically if we add an external spaceperiodic potential to Eq. (14). Such a system is in general nonintegrable, and we may expect the additional symmetry (21) to be absent. To be precise, we choose the following model here:

$$
H=-\cos p-\frac{1}{2} \cos (2 p)-\frac{\xi^{2}}{3} \cos \left(\frac{x}{\xi}\right)-\frac{\xi}{3} x E(t) .
$$

The corresponding equations of motion read

$$
\begin{gathered}
\dot{x}=\sin p+\sin (2 p), \\
\dot{p}=-\frac{\xi}{3} \sin \left(\frac{x}{\xi}\right)+\frac{\xi}{3} E(t) .
\end{gathered}
$$

The free parameter $\xi$ can be used to reach the limit of Eq. (9). Indeed for $\xi \rightarrow 0, \dot{p} \rightarrow 0$. Choosing a small value of $|p|$ $\ll 1$ will keep the momentum small. This allows for an expansion of the right hand side of Eq. (24) to first order. Additional rescaling $x \rightarrow x / \xi$ and $p \rightarrow(3 / \xi) p$ transforms the problem exactly to Eq. (9) (with $\gamma=0$ ). On the other side, a choice of a finite value of $p$ may lead to trajectories that are not contained in Eq. (9). In addition, Eq. (23) is periodic in $p$, so the phase space is compact.

Let us discuss the relevant symmetries of Eqs. (24) and (25). Due to the presence of the $\cos (2 p)$ term in Eq. (23) the

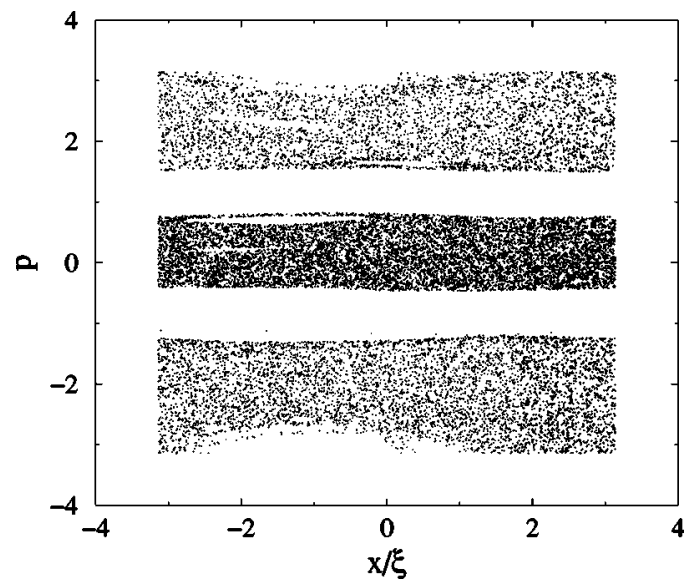

FIG. 7. Poincaré map of Eqs. (24) and (25) for $\xi=0.35$.

shift symmetry of the kinetic energy is broken (in terms of electrons this implies loss of the particle-hole symmetry). We can identify the following symmetry operations which lead to a change of sign of $\dot{x}$ :

$$
\begin{gathered}
x \rightarrow-x, p \rightarrow-p, t \rightarrow t+\frac{T}{2} \text { if }\left\{E_{s h}\right\}, \\
t \rightarrow-t, p \rightarrow-p \text { if }\left\{E_{s}\right\} .
\end{gathered}
$$

In the following we choose $E(t)$ from Eq. (11) which ensures that both symmetries are broken.

For $\xi \ll 1$ the phase space of Eq. (23) is characterized by the presence of two disconnected stochastic layers. One of them corresponds to the layer in Fig. 1. In Fig. 7 we show the Poincaré map of Eqs. (24) and (25) for $\xi=0.35$. Note that the central layer is the one which continuously transforms into the layer in Fig. 1 when $\xi \rightarrow 0$. An increasing of $\xi$ will lead to a merging of the layers, which is followed by losses (closings) of ballistic channels that are located near the boundaries of the central stochastic layer. The Poincare map for $\xi=1$ is shown in Fig. 8 .

Let us discuss a trajectory with initial conditions $p=0$ and $x=\xi \pi$, which ensures that we always start in the stochastic layer which corresponds to Fig. 1 in the limit of small

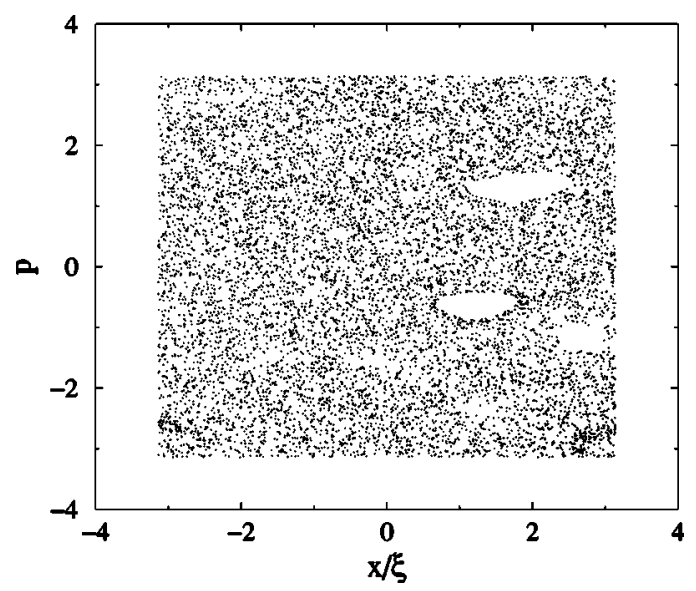

FIG. 8. Poincaré map of Eqs. (24) and (25) for $\xi=1$. 


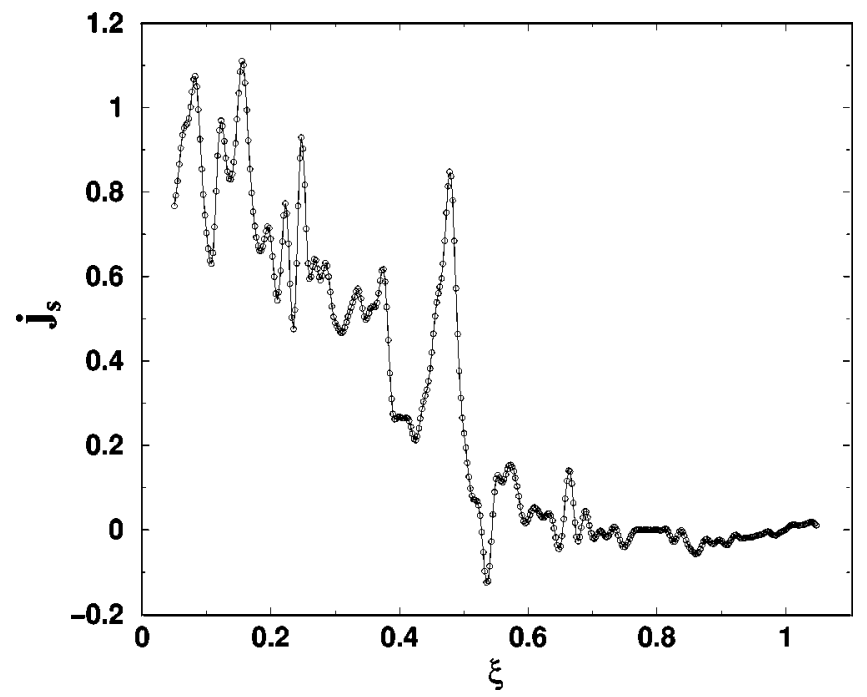

FIG. 9. $j_{s}$ as a function of $\xi$. Note that the error in determining the current value is of the order of $10 \%$. A fine structure of pronounced peaks is connected with the opening of new ballistic channels due to the overlap of the stochastic layer with high-order resonances.

$\xi$. The dependence of the scaled dc current $j_{s}=\langle\dot{x}\rangle / \xi$ is shown in Fig. 9 as a function of $\xi$. Specifically its values for the presented Poincaré maps are $j_{s} \approx 0.63$ for $\xi=0.35$ and $j_{s} \approx 0.002$ for $\xi=1$. We observe indeed that the current variations correspond to a closing or opening of ballistic channels.

The above results support the general expectation that in the Hamiltonian limit a mixed phase space is needed, so that the current vanishes both in the case of an integrable system as well as in the case of a fully chaotic one.

\section{CONCLUSIONS}

We presented a symmetry approach to the effect of rectification due to external ac fields applied to a lowdimensional dynamical system with optional contact to a heat bath. The nonlinear (nonadiabatic) response is used to explain the effect. We explained the mechanisms of such a rectification for different cases. In the case of a particle moving in a space-periodic potential the explanation is given in terms of desymmetrization of ballistic channels which correspond to motion in different directions. While this explanation starts from the case of a nondissipative Hamiltonian limit, we showed that the ballistic channels are robust with respect to adding dissipation and fluctuations. The mechanism of the directed current in the presence of dissipation is hidden in the desymmetrization of the basins of attraction of previously symmetry-related limit cycles with oppositely directed velocities.

A recent geometric approach by Schanz et al. in Ref. [16] provides an elegant way to account for the average drift velocity in the Hamiltonian case. The basic ingredient of this approach is the assumption of an invariant density distribution in a stochastic layer being constant inside the layer. The resulting sum rule, when evaluated for concrete cases, pro- vides average velocities that quantitatively agree with our observations. A consequence of the sum rule is that a nonzero directed current may appear only in systems with a mixed phase space. This result correlates with our discussion of the mechanisms of directed currents in terms of ballistic channels, which may appear only if the stochastic layer has some bounds with regular phase space regions, and the ballistic channels occur precisely in the neighborhood of these bounds. Open questions are the following (i) Is there purely diffusive (chaotic) directed motion besides transport through ballistic channels? (ii) When can one of these two mechanisms prevail?

It was shown that the symmetry properties of the FokkerPlanck equation match the symmetries of the corresponding deterministic equations of motion in the absence of noise. Similar results hold for a Boltzmann equation [3].

Our symmetry considerations may be as well used to explain rectifications in such diverse situations as (i) the directed motion of particles in non-Newtonian liquids [17], (ii) the appearance of ring currents for particles moving in a two-dimensional space-periodic potential $[18,19]$, (iii) the appearance of directed heat flux currents in systems of interacting particles [4], (iv) the appearance of a nonzero dc polarization, (v) quantum ratchets [10], to name a few. Symmetry analysis is also instructive in the case of less conventional models, like systems with built-in asymmetry [20-22], where the asymmetry is hidden in a many body system's internal interactions.

\section{ACKNOWLEDGMENTS}

We thank T. Dittrich, M. V. Fistul, P. Hänggi, R. Ketzmerick, A. Miroshnichenko, P. Reimann, and H. Schantz for stimulating discussions, and A. Miroshnichenko for numerical help with solving Fokker-Planck equations.

\section{APPENDIX:}

The Fokker-Planck equation for the probability distribution $W(x, p, t)$ with $p \equiv \dot{x}$ of Eqs. (7),(8) reads [1]

$$
\frac{\partial W}{\partial t}=-p \frac{\partial W}{\partial x}+\frac{1}{m} \frac{\partial}{\partial p}\left[-f(x)-E(t)+\gamma p+\frac{\gamma}{m \beta} \frac{\partial}{\partial p}\right] W .
$$

This equation is linear in $W$, preserves the norm $\int W d x d p$, and is dissipative. For a fixed norm any initial condition will converge to a single attractor solution $W_{s}$. For the case $E(t)=0$ it is easy to see that the attractor is the Gibbs distribution. For nonzero $E(t)$ the attractor solution $W_{s}$ will be periodic in $x$ and $t$. The average current is given by

$$
\langle p\rangle=\int p W_{s} d x d p d t
$$

It follows that Eq. (A1) is invariant under the following transformations:

$$
\hat{S}_{a}, x \rightarrow-x, p \rightarrow-p, t \rightarrow t+\frac{T}{2} \text { if }\left\{f_{a}, E_{s h}\right\},
$$




$$
\hat{S}_{b}, x \rightarrow x, t \rightarrow-t, p \rightarrow-p \text { if }\left\{E_{s}, \quad \gamma=0\right\} .
$$

At the same time Eq. (A2) is changing sign. Since the solution $W_{s}$ is unique, the conclusion is that $\langle p\rangle=0$.

The overdamped limit $m=0$ is described by the following Fokker-Planck equation [1]:

$$
\gamma \frac{\partial W}{\partial t}=\frac{\partial}{\partial x}[-f(x)-E(t)] W+\frac{1}{\beta} \frac{\partial^{2} W}{\partial x^{2}} .
$$

The average current is given by

$$
\langle p\rangle=\int f(x) W_{s} d x d t .
$$

The symmetry $\hat{S}_{a}$ again holds. However, the symmetry

$$
\hat{S}_{c}, x \rightarrow x+\frac{\lambda}{2}, t \rightarrow-t \text { if }\left\{f_{s h}, E_{a}, m=0\right\}
$$

does not follow from Eq. (A4) in a similar way. It is interesting to note that Reimann [23] considered the symmetries of the original stochastic differential equations (7),(8) and argued that $\hat{S}_{c}$ holds for nonzero noise intensities. Fistul [24] showed that $\hat{S}_{c}$ connects the original Fokker-Planck equation (A4) with its conjugated counterpart.

Here we will use a related approach and prove the vanishing of Eq. (A5) when $\hat{S}_{c}$ holds. First we remind that the distribution $W_{s}$ is periodic in $x$ and $t$. Next we note that the operators $\partial / \partial t$ and $\partial / \partial x$ are anti-Hermitian on the space of $x, t$-periodic functions. Let us define the operator $T$ as

$$
T=\frac{-\frac{\partial}{\partial x}}{\gamma \frac{\partial}{\partial t}-\frac{\partial^{2}}{\beta \partial x^{2}}+E(t) \frac{\partial}{\partial x}} .
$$

Then Eq. (A4) can be rewritten as a Lippmann-Schwinger type integral equation,

$$
W=1+T f(x) W .
$$

Provided the conditions for $\hat{S}_{c}$ hold, i.e., $E(t) \equiv E_{a}(t)$, the operator $T$ has the property

$$
T^{\dagger}=-T(-t), T\left(x+x_{0}\right)=T(x) .
$$

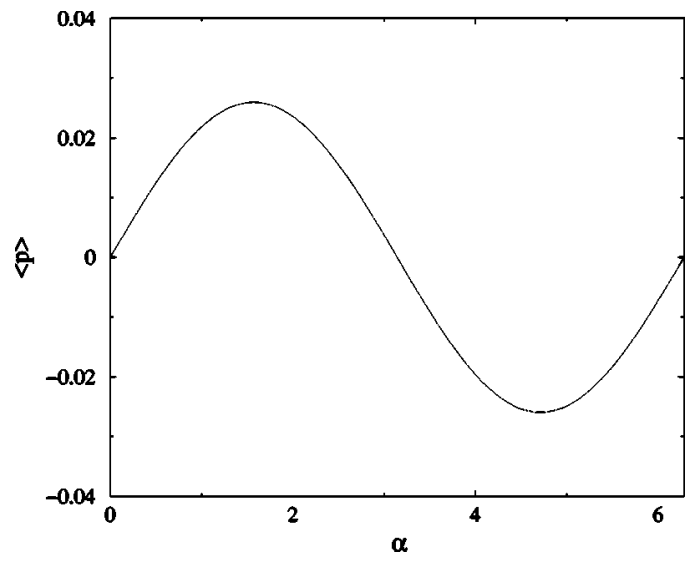

FIG. 10. $\langle p\rangle$ as a function of $\alpha$ (see text).

Expanding Eq. (A7) in a formal series in $f(x)$ we obtain

$$
W_{s}=1+\sum_{n=1}^{\infty}[T f(x)]^{n} .
$$

With Eq. (A5) we obtain for the average current

$$
\langle p\rangle=\sum_{n=1}^{\infty} \int f(x)[T f(x)]^{n} d x d t .
$$

Since all terms in Eq. (A10) are real valued, we conclude that all integrals with even $n$ vanish because of $f(x)$ $\equiv f_{s h}(x)$ and these terms contain odd powers of $f$. All integrals with odd $n$ vanish because of Eq. (A8), since they contain odd powers of $T$. Thus we find that indeed the average current exactly vanishes when $\hat{S}_{c}$ holds.

We also performed a numerical solution of Eq. (A4) for $T=\gamma=1$ and $f(x)=\sin x, E(t)=\sin (\omega t)+\sin (2 \omega t+\alpha)$ with $\omega=0.8$. The function $W$ was expanded in a Fourier series in $x$ and twenty Fourier harmonics have been taken into account. The resulting coupled ordinary differential equations were simply integrated until the system reached the final attractor $W_{s}$.

For the above choice of functions $f(x)$ and $E(t)$ the symmetry $\hat{S}_{a}$ is violated because $E(t)$ is not shift symmetric. At the same time the symmetry $\hat{S}_{c}$ is violated for all values of $\alpha$ except for $\alpha=0, \pm \pi, \pm 2 \pi, \ldots$ In Fig. 10 the dependence of the average current $\langle p\rangle$ (A5) on $\alpha$ is shown. We observe that for $\alpha=0, \pi, 2 \pi$ the symmetry $\hat{S}_{c}$ is restored and the current is vanishing (the absolute numerical value is less than $\left.10^{-12}\right)$.
[1] P. Reimann, Phys. Rep. 361, 57 (2002).

[2] S. Flach, O. Yevtushenko, and Y. Zolotaryuk, Phys. Rev. Lett. 84, 2358 (2000).

[3] O. Yevtushenko, S. Flach, Y. Zolotaryuk, and A.A. Ovchinnikov, Europhys. Lett. 54, 141 (2001).

[4] S. Flach, Y. Zolotaryuk, A. Miroshnichenko, and M. V. Fistul, Phys. Rev. Lett. 88, 184101 (2002).

[5] S. Flach and A.A. Ovchinnikov, Physica A 292, 268 (2001); S.
Flach, A. Miroshnichenko, and A.A. Ovchinnikov, Phys. Rev. B 65, 104438 (2002).

[6] A.A. Ovchinnikov, e-print cond-mat/0110616.

[7] Underdamped systems often display hysteretic dependence on external fields, which leads to multivalued response functions. In that case the overall symmetry of a response function is not sufficient to conclude about the current rectification. The case of Eqs. (9) and (11) in the adiabatic limit is a nice example. 
For $\alpha=\pi / 2$ the ac drive is antisymmetric, yet a nonzero rectification will take place, in full accord with the symmetry analysis of the underlying equations of motion.

[8] R. Bartussek, P. Hänggi, and J.G. Kissner, Europhys. Lett. 28, 459 (1994).

[9] M. V. Fistul, Phys. Rev. E 65, 046621 (2002).

[10] S. Scheidl and V.M. Vinokur, e-print cond-mat/0201008.

[11] G. M. Zaslavsky, Physics of Chaos in Hamiltonian Systems (Imperial College Press, London, 1998).

[12] S. Denisov and S. Flach, Phys. Rev. E 64, 056236 (2001); S. Denisov, J. Klafter, M. Urbakh, and S. Flach, Physica D 170, 131 (2001).

[13] J. Mateos, Phys. Rev. Lett. 84, 258 (2000).

[14] O. Yevtushenko, S. Flach, and K. Richter, Phys. Rev. E 61, 7215 (2000).
[15] M. Vavilov, V. Ambegaokar, and I. Aleiner, Phys. Rev. B 63, 195313 (2001).

[16] H. Schanz, M.-F. Otto, R. Ketzmerick, and T. Dittrich, Phys. Rev. Lett. 87, 070601 (2001).

[17] A.K. Vidybida and A.A. Serikov, Phys. Lett. 108A, 170 (1985).

[18] A.W. Ghosh and S.V. Khare, Phys. Rev. Lett. 84, 5243 (2000).

[19] D. del-Castillo-Negrete, Phys. Fluids 10, 576 (1998).

[20] S. Denisov, Phys. Lett. A 296, 197 (2002).

[21] Z. Zheng, G. Hu, and B. Hu, Phys. Rev. Lett. 86, 2273 (2001).

[22] S. Cilla, F. Falo, and L.M. Floria, Phys. Rev. E 63, 031110 (2001).

[23] P. Reimann, Phys. Rev. Lett. 86, 4992 (2001).

[24] M. V. Fistul (private communication). 